\title{
Leucine-Rich Repeat Genes and the Fine-Tuning of Synapses
}

\section{Clyde Francks}

$\mathrm{P}$ resynaptic neurexins and postsynaptic neuroligins are celladhesion proteins that interact across the synaptic cleft and influence synaptic function (1). These proteins have received recent attention in psychiatry because of genetic evidence linking them to schizophrenia, autism, and mental retardation (1). However, during the last 2 years, an additional family of synaptic adhesion proteins has been found to play a directly cooperative role with the neurexins and neuroligins in glutamate synapse differentiation (2-4) (Figure 1). This novel gene family is known as the leucine-rich repeat transmembrane neuronal (LRRTM) family, and early evidence is accumulating that these genes are also implicated in psychiatric disease (5-8).

The genetic evidence implicating the neurexins and neuroligins in psychiatry is based particularly on rare and overtly disruptive alterations in humans, such as copy number variants and mutations, as well as on animal models (1). The specific abundances of each neurexin/neuroligin family member or their isoforms that interact at an individual synapse are likely to affect individual synaptic transmission functions and stability $(1,9)$. Thus, the properties of neural networks and systems important for cognition can easily be imagined to depend on the precise functioning of these synaptic organizer/modifier genes.

The four-member LRRTM gene family was first described in 2003 (10). The LRRTM proteins have intracellular tails of 71 or 72 amino acids, single transmembrane domains, and 10 extracellular leucinerich repeat (LRR) domains (Figure 1). The LRR domains are involved in protein-protein interactions and are found in a variety of brainenriched, neuronal growth-modulating genes (10). The structures and expression distributions of LRRTMs point to functions in cell surface signaling and/or cell adhesion, particularly within postmitotic neurons of specific regions of the brain $(5,10)$. The LRRTM messenger RNAs are developmentally upregulated in the mouse brain, starting from at least as early as embryonic day $13-15$, and reach high levels by postnatal day $1(10)$. In the adult mouse brain, LRRTM1 and LRRTM2 are both expressed strongly in neurons of the cerebral cortex, striatum, CA1-CA3 pyramidal layers, and the dentate gyrus granular layer of the hippocampus and broadly in the thalamus (as well as in the olfactory bulb) (10). However, although LRRTM1 is expressed in superficial neurons of the cerebral cortex excluding Layer 1, LRRTM2 expression is more prominent in deep layers. Also LRRTM1 is not expressed strongly in the cerebellum, in contrast to LRRTM2.

The other family members, LRRTM3 and LRRTM4, also show similarities of expression in the mouse brain, including high expression in the cerebral cortex, with a similar pattern across the layers (i.e., excluding Layer 1; high in Layer 2; lower in Layer 3; and moderate in Layers 4, 5, and 6) (10). Both genes are also expressed highly in the striatum, whereas in the hippocampus high expression of LRRTM3 and LRRTM4 is seen in the dentate gyrus granular layer, but

From the Max Planck Institute for Psycholinguistics, Nijmegen, The Netherlands.

Address correspondence to Clyde Francks, MA DPhil, Max Planck Institute for Psycholinguistics, Wundtlaan 1,6525 XD Nijmegen, The Netherlands; E-mail: clyde.francks@mpi.nl.

Received Sep 28, 2010; revised Dec 17, 2010; accepted Dec 17, 2010.

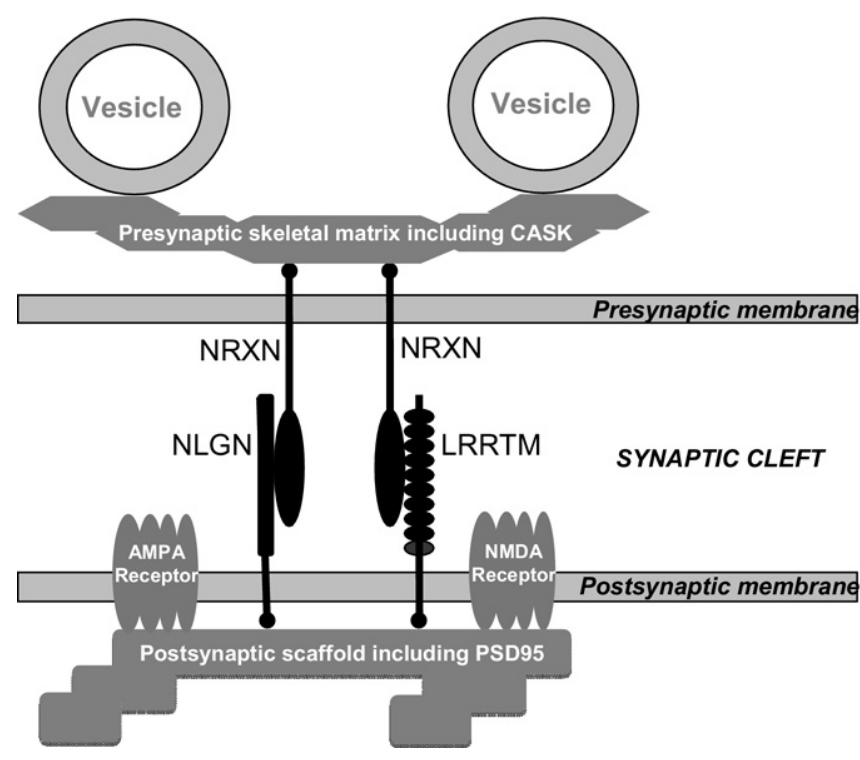

Figure 1. Representation of neurexins (NRXN), neuroligins (NLGN), and leucine-rich repeat transmembrane neuronal (LRRTM) genes interacting across the glutamate synapse. The absolute and relative abundances of these proteins and their isoforms are thought to specify a functional code that affects individual synapse strength and specificity. All three gene families are now implicated in psychiatric phenotypes by genetic studies. CASK, Ca-calmodulin-dependent serine protein kinase, AMPA, $\alpha$-amino-3-hydroxy-5-methylisoxazole propionate; NMDA, N-methyl-D-aspartate; PSD95, post-synaptic density-95.

only LRRTM4 is seen in neurons of the CA3 layer. Neither LRRTM3 nor LRRTM4 are expressed strongly in the thalamus, in contrast to LRRTM1 and LRRTM2 (10). These region-specific expression patterns are broadly mirrored in expression profiling of adult human brain (e.g., Allen Brain Atlas; http://human.brain-map.org) as well as in situ hybridization in human fetal brain for LRRTM1 (5). Taken together, the structural similarities and expression patterns of the LRRTMs point to related but partly unique roles, so that functional redundancy between at least some of the members is a clear possibility.

Francks et al. (5) first implicated LRRTMs in human behavioral variability and psychiatry. They initially found a parent-of-origin effect of chromosome $2 \mathrm{p} 12$ on handedness, within dyslexic siblings. LRRTM1 was a neuronal candidate gene on $2 \mathrm{p} 12$ and showed variable maternal imprinting of messenger RNA expression in cell lines and brain tissue (5) (imprinted genes are a small minority of all genes that are regulated-by epigenetic alterations - to be differently active, depending on parent-of-origin). There was also inconsistent evidence for paternal-specific association-with handedness-of a haplotype upstream of LRRTM1, although it was hypothesized that much of the $2 p 12$ effect might be caused by epigenetic abnormalities of imprinting (5). Francks et al. (5) also observed a paternal effect of the LRRTM1 locus in familial schizophrenia, with the same associated haplotype as implicated in handedness. This finding has recently received further support (7). 
Working from a different direction, Linhoff et al. (4) used a fibroblast-neuron coculture assay to screen a genome-wide complementary DNA library for novel synaptogenic proteins and identified LRRTM1 as a potent inducer of excitatory synaptogenesis. They showed that LRRTM1 instructs excitatory presynaptic differentiation and the development of functional glutamate release sites, mediates excitatory postsynaptic differentiation, and localizes to excitatory postsynaptic sites. A mouse knockout of LRRTM1 revealed a subtle effect on the distribution of the vesicular glutamate transporter VGLUT1 in vivo (4). However, a degree of functional redundancy between LRRTMs, particularly between LRRTM1 and LRRTM2, might prevent a more overt phenotype in the LRRTM1 knockout (4).

Major steps forward have followed during the last year. Independent studies have now shown that LRRTM2, like the neuroligins, binds directly with neurexins to regulate glutamate synaptogenesis and influence synapse signaling $(2,3)$, presumably by interacting across the synaptic cleft (Figure 1). Moreover, whereas neuroligins bind to neurexins containing or lacking an insert in splice site \#4, LRRTM2 binds only to neurexins that lack an insert here (3). These data identify the LRRTM proteins as key players in a molecular ensemble at the synapse that is already known to be disrupted in some cases of psychiatric disease, and in which the LRRTMs themselves are likely to play a part in the encoding of individualized synaptic properties. Considered together, the neurobiological studies point to a role of the LRRTMs in the "fine-tuning" of synapses rather than being individually essential for synaptic formation. It seems unsurprising that disruption of such fine-tuning genes might result in psychiatric phenotypes, in which subtle functional abnormalities generally manifest in the absence of major morphological changes.

The potential involvement of LRRTM1 in human handedness and schizophrenia suggests a role in the development and/or maintenance of lateralized cerebral functions, which are sometimes subtly disrupted in several neuropsychiatric conditions (5). Many highlevel cognitive functions, such as language, are lateralized in the human brain (5). No obvious asymmetries in brain expression of LRRTM1 have been observed in the few developmental stages that have been analyzed to date, in mice or humans (5). A possibility is that LRRTM1 expression responds only subtly to other pre-established asymmetrical signals, to consolidate the formation and maintenance of neuronal circuitry that supports lateralized functions. Regardless of asymmetry, there are other indications for how LRRTM dysfunction might ultimately lead to psychiatric pathology. For example, de Wit et al. (2) observed that in vivo knockdown of LRRTM2 decreases the strength of glutamatergic synaptic transmission (without affecting presynaptic properties). Also, the apparent role of LRRTMs in synaptic stability points to a possible function in synaptic pruning (the selective loss of synapses) which might be pathogenically accelerated in schizophrenia.

Evidence has also emerged linking LRRTM3 to psychiatric and potentially neurological dysfunction. Majercak et al. (7) performed high-throughput small interfering RNA screening of 15,200 genes and identified LRRTM3 as a neuronal gene that promotes the processing of amyloid precursor protein (APP) by the protease $\beta$-site APP-cleaving enzyme (BACE), while noting that LRRTM3 maps to a region of chromosome 10 linked to Alzheimer's disease. Some familial forms of Alzheimer's disease are thought to be caused by elevated proteolytic production of the A $\beta 42$ peptide from APP (7). Polymorphisms in LRRTM3 were also associated with autism spectrum disorder in a recent genetic study (8). The broad range of central nervous system phenotypes potentially linked to LRRTM genes therefore mirrors the wide phenotypic spectrum that is associated with the neurexins and neuroligins (1).

The genes that encode LRRTM1, LRRTM2, and LRRTM3 are nested within introns of $\alpha-\mathrm{N}$-catenin genes in the mammalian genome $(5,10)$. This arrangement might make it unlikely to find psychiatric patients with gross genomic copy number abnormalities affecting the LRRTM genes, because the surrounding $\alpha-\mathrm{N}$-catenin genes would presumably also be disrupted, and the resulting compound phenotypes possibly would be severe or lethal. Nonetheless, given the clear genetic evidence implicating the neurexins and neuroligins in psychiatry, further research in this area must surely also consider the LRRTMs, whose functions are now known to be so closely related.

Future research priorities include further human genetic and epigenetic studies of the LRRTM gene family in psychiatric and behavioral phenotypes, including the possibility for effects mediated partly via the surrounding $\alpha-\mathrm{N}$-catenin genes. A deeper characterization of all the LRRTM family members in model organisms is warranted to better understand how functionally related these genes truly are. Other questions include whether any LRRTMs have critical rather than only modifying roles in synaptogenesis and signaling; whether any LRRTMs affect inhibitory as well as excitatory transmission (the neurexins and neuroligins have roles in both) (9); and what are the effects of targeted interference with LRRTM functions on learning, memory, and plasticity.

This work was supported by the Max Planck Institute for Psycholinguistics, Nijmegen, the Netherlands. The author has previously been an employee in the pharmaceutical industry and has held stocks and options but reports no potential conflicts of interest relating to this article.

1. Sudhof TC (2008): Neuroligins and neurexins link synaptic function to cognitive disease. Nature 455:903-911.

2. de Wit J, Sylwestrak E, O'Sullivan ML, Otto S, Tiglio K, Savas JN, et al. (2009): LRRTM2 interacts with neurexin 1 and regulates excitatory synapse formation. Neuron 64:799-806.

3. Ko J, Fuccillo MV, Malenka RC, Südhof TC (2009): LRRTM2 functions as a neurexin ligand in promoting excitatory synapse formation. Neuron 64:791-798.

4. Linhoff M, Lauren J, Cassidy R, Dobie F, Takahashi H, Nygaard H, et al. (2009): An unbiased expression screen for synaptogenic proteins identifies the LRRTM protein family as synaptic organizers. Neuron 61:734749.

5. Francks C, Maegawa S, Lauren J, Abrahams B, Velayos-Baeza A, Medland $\mathrm{S}$, et al. (2007): LRRTM1 on chromosome $2 \mathrm{p} 12$ is a maternally suppressed gene that is associated paternally with handedness and schizophrenia. Mol Psychiatry 12:1129-1139.

6. Ludwig KU, Mattheisen M, Muhleisen TW, Roeske D, Schmal C, Breuer R, et al. (2009): Supporting evidence for LRRTM1 imprinting effects in schizophrenia. Mol Psychiatry 14:743-745.

7. Majercak J, Ray W, Espeseth A, Simon A, Shi X, Wolffe C, et al. (2006): LRRTM3 promotes processing of amyloid-precursor protein by BACE1 and is a positional candidate gene for late-onset Alzheimer's disease. Proc Natl Acad Sci U S A 103:17967-17972.

8. Sousa I, Clark T, Holt R, Pagnamenta A, Mulder E, Minderaa R, et al. (2010): Polymorphisms in leucine-rich repeat genes are associated with autism spectrum disorder susceptibility in populations of European ancestry. Mol Autism 1:7.

9. Zhang C, Atasoy D, Araç D, Yang X, Fucillo MV, Robison AJ, et al. (2010): Neurexins physically and functionally interact with GABAA receptors. Neuron 66:403-416.

10. Lauren J, Airaksinen M, Saarma M, Timmusk T (2003): A novel gene family encoding leucine-rich repeat transmembrane proteins differentially expressed in the nervous system. Genomics 81:411-421. 